\title{
Pure T3 thyrotoxicosis from a Struma Ovarii characterised by a paradoxical rise in thyroxine on treatment
}

\author{
James Prentice ${ }^{1}$, Kate Panter², Ayoma Attygalle ${ }^{3}$, Thomas Ind ${ }^{3}$ and Malcolm Prentice 4 \\ 'University of Glasgow Medical School, Glasgow, UK, 2Department of Gynaecology, Guy's Hospital, London, UK, \\ ${ }^{3}$ Royal Marsden Hospital, London, UK, and ${ }^{4}$ Department of Endocrinology, Croydon University Hospital, London, UK
}

Correspondence should be addressed to M Prentice

Email

malcolmprentice@nhs.net

\section{Summary}

A 33-year-old female presented with a right $11.6 \mathrm{~cm}$ ovarian cyst. Routine pre-operative thyroid function tests showed thyroid stimulating hormone (TSH) of less than $0.02 \mathrm{mU} / \mathrm{L}$ (0.3-3.05) and a free thyroxine (FT4) of $5.5 \mathrm{pmol} / \mathrm{L}$ (10-28.2) suggesting either assay interference, triiodothyronine (T3) ingestion or hypopituitary hypothyroidism. A free triiodothyronine (FT3) level was requested which was high normal $6.9 \mathrm{pmol} / \mathrm{L}$ (3.1-8.1). Parallel assays on a different platform were similar but with a raised FT3 of $7.2 \mathrm{pmol} / \mathrm{L}$ (3.1-6.8). TSH receptor stimulating antibody (TSHAb) and thyroid peroxidase antibodies (TPO) were negative. Antithyroglobulin antibody (TgAb) was positive at $155.6 \mathrm{IU} / \mathrm{mL}(0-115)$. She was clinically euthyroid. Thyroid ultrasound showed a normal sized mildly heterogeneous gland with low blood flow and a solitary $1.5 \mathrm{~cm}$ U3 (BTA) nodule with higher blood flow. Thyroid TC ${ }^{99 m}$ uptake was very low $0.2 \%$ (0.6-3.0) with no nodule uptake. These results demonstrated an extrathyroidal source of excessive autonomous T3 production resulting in the low thyroxine (T4). With carbimazole her TSH rose to $11.9 \mathrm{mU} / \mathrm{L}$, FT4 rose to $7.7 \mathrm{pmol} / \mathrm{L}$ and FT3 reduced to $3.6 \mathrm{pmol} / \mathrm{L}$. Histological diagnosis was Struma Ovarii. Her TSH, FT4 and FT3 remained normal thereafter. In conclusion, an extrathyroidal source of high T3 secretion was diagnosed using routine thyroid tests and scans. We believe this is the first description of a Struma Ovarii exclusively secreting T3 hormone characterised by the paradoxical rise of a low FT4 to normal with treatment. Two years later she developed non-secreting peritoneal deposits of highly differentiated follicular carcinoma.

\section{Learning points:}

- Abnormally low TSH and FT4 levels suggestive of possible T3 ingestion, or less likely, hypopituitary hypothyroidism should always be followed by an assay of FT3.

- The diagnosis of an extrathyroidal source of T3 can be made using conventional thyroid tests, thyroid ultrasound scanning and technetium thyroid uptake and scan imaging. In a pre-menopausal patient this avoids a radiation dose to the pelvis.

- Pelvic radioisotope scanning of a suspected Struma Ovarii causing thyrotoxicosis can be reserved for patients whose thyroid function remains abnormal after initial surgery.

- Carbimazole is effective in the treatment of extrathyroidal autonomous T3 hormone production from a Struma Ovarii.

- The pathological appearance of a Struma Ovarii is not a guide to its malignancy. Even with a benign appearance they can disseminate to peritoneum, as highly differentiated follicular carcinoma (previously known as peritoneal strumosis).

- Hyperthyroid secretion by a Struma Ovarii may not be replicated in the metastatic follicular carcinoma in the peritoneum. 


\section{Background}

Struma Ovarii is the presence of thyroid tissue as a major cellular component in an ovarian tumour. To date, the secretion of thyroid hormones $\mathrm{T} 3$ and $\mathrm{T} 4$ by an ovarian Struma Ovarii tumour is a recognised rare cause of thyrotoxicosis (1) and occurs in about $8 \%$ of patients with Struma Ovarii (2). The thyrotoxicosis from a Struma Ovarii characteristically is caused by both raised $\mathrm{T} 3$ and raised $\mathrm{T} 4$ secretion $(1,2)$. Treatment with antithyroid medication and surgical removal invariably results in the reduction of both thyroid hormones levels into their normal ranges. One previous case of $\mathrm{T} 3$ toxicosis secretion from Struma Ovarii has been described; however, the FT4 levels in that case remained in the normal range before, during and after surgery (3). We describe a first presentation, diagnosis and management of a Struma Ovarii which exclusively secreted T3. The TSH was totally suppressed by the high T3, and $\mathrm{T} 4$ was very low. There was no evidence of $\mathrm{T} 3$ ingestion or pituitary hypothyroidism and assay interference was excluded. Routine thyroid antibody tests, ultrasound and technetium scans pointed to an extrathyroidal source of autonomous T3 production likely to be from the ovary. There was no clinical indication for a radioisotope pelvic scan, as urgent surgery was planned and if successful in removing the source of $\mathrm{T} 3$ production will have spared the patient a dose of radiation to the pelvis. Pre-operative carbimazole therapy resulted in increasing $\mathrm{T} 4$ levels following the expected reduction of $\mathrm{T} 3$ and the resulting gradual increase of TSH. This unique paradoxical rise of FT4 with preoperative carbimazole treatment and surgery confirmed the absence of $\mathrm{T} 4$ secretion from the Struma tumour and confirmed that the T3 secretion was extrathyroidal. T3 secretion from the Struma Ovarii was confirmed by the histology and the surgical removal which resulted in the complete restoration of normal thyroid function. There is evidence of expression of type II iodothyronine deiodinase (DIO2) in ovarian epithelial cells (4) and tumours (5) as a possible cause of the T3 secretion by the tumour resulting in $\mathrm{T} 4$ conversion to T3. Two years later the patient developed the well-recognised dissemination with a peritoneal highly differentiated follicular carcinoma.

\section{Case presentation}

A 33-year-old nulliparous female who was planning conception presented with a 1-year history of intermittent left iliac fossa pain and gradual onset of feeling hot, sweating and weight loss. Ultrasound had showed a right ovarian dermoid cyst which had increased from $4.6 \mathrm{~cm}$ to

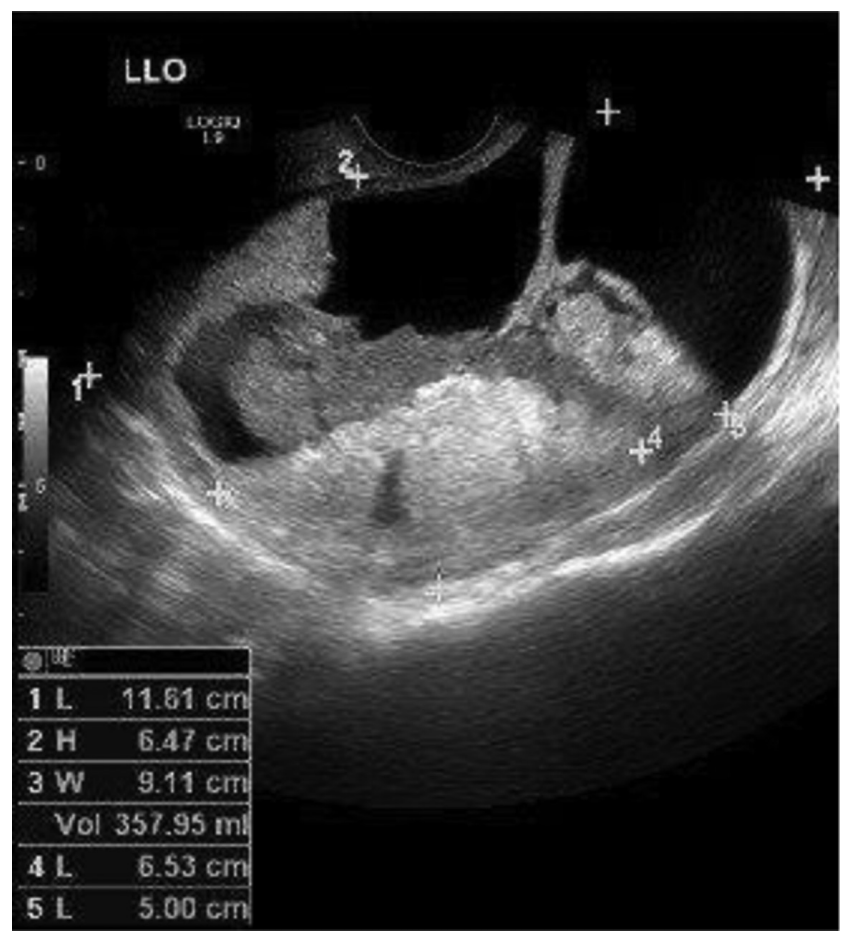

Figure 1

Pelvic ultrasound showing the right ovarian cyst.

$11.6 \mathrm{~cm}$ diameter over 4 years (Fig. 1). Pre-op tests showed low-risk ROMA and minimally elevated CA 19-9. She was referred for urgent surgery. Routine pre-operative blood tests showed an undetectable TSH of $<0.01 \mathrm{mU} / \mathrm{L}(0.27-4.2)$ and a FT4 of $5.5 \mathrm{pmol} / \mathrm{L}$ (10-28.2). Her past history was a 4-year history of acne, hirsutism and oligomenorrhoea from polycystic ovaries and had had dermal syringomas on the skin removed with laser therapy. There was no past history or family history of thyroid disease. There were no symptoms of drug or T3 abuse, pituitary disease, head injury or visual disturbance. She had a high exercise regimen completing several half marathons. She was a non-smoker and drank little alcohol and ate a normal diet. She had a copper IUD and was taking a combined ethinyloestradiol and cyproterone acetate oral contraceptive for her acne and hirsutism. On examination, she had a BMI of 20.3 at $61.4 \mathrm{~kg}$. She was clinically euthyroid, pulse 80 in sinus rhythm and blood pressure was 100/68. There were no signs of thyroid acropachy, and thyroid clinical examination was normal. Visual fields were full with no features of pituitary disease or virilisation.

\section{Investigation}

Routine pre-operative thyroid function tests showed TSH was less than $0.02 \mathrm{mU} / \mathrm{L}(0.27-4.2)$ with an FT4 of 
Table 1 Comparison of thyroid hormones in a pre-treatment sample assayed in Ortho Vitros 5600 and Roche cobas Elecsys assays.

\begin{tabular}{|c|c|c|c|c|}
\hline & \multicolumn{2}{|c|}{ Ortho assay } & \multicolumn{2}{|c|}{ Roche assay } \\
\hline & Normal range & Value & Normal range & Value \\
\hline $\mathrm{TSH} \mathrm{mU} / \mathrm{L}$ & $0.3-3.05$ & $<0.01$ & $0.27-4.2$ & 0.06 \\
\hline FT4 pmol/L & $10-28$ & 5.5 & $10.8-25.5$ & 7.17 \\
\hline FT3 pmol/L & $4.3-8.1$ & 6.9 & $3.1-6.8$ & 7.2 \\
\hline
\end{tabular}

$5.5 \mathrm{pmol} / \mathrm{L}$ (10-28 Ortho assay). These results suggested either T3 ingestion, hypopituitary hypothyroidism or assay interference with either of the assays. In addition, therefore FT3 was assayed which was high normal at 6.9 $\mathrm{pmol} / \mathrm{L}$ (4.3-8.1 Ortho assay). All the assays were repeated on the same sample on a different assay platform with similar results, except that the T3 was in the thyrotoxic range at $7.2 \mathrm{pmol} / \mathrm{L}$ (3.1-6.8 Roche assay) see Table 1 . Pituitary function tests were normal. TSHAb was negative at $0.39 \mathrm{IU} / \mathrm{mL}(0-0.81)$, TPO was negative at $28.4 \mathrm{IU} /$ $\mathrm{mL}(0-34)$ and $\mathrm{TgAb}$ was positive $155.6 \mathrm{IU} / \mathrm{mL}(0-115)$. Thyroglobulin was $35.1 \mathrm{mg} / \mathrm{L} \quad(11.3-28.9)$. Thyroid ultrasound showed a normal sized gland with a solitary $1.5 \mathrm{~cm} \mathrm{U3} \mathrm{(BTA} \mathrm{classification)} \mathrm{nodule} \mathrm{in} \mathrm{the} \mathrm{right} \mathrm{lobe}$ (Fig. 2), with a relatively high blood flow compared with the rest of the gland which had very low blood flow on doppler throughout with a mildly heterogeneous echo pattern. Ultrasound guided fine needle aspiration (FNA) of the nodule showed benign hyperplastic appearance classified Thy2 (BTA classification). Thyroid technetium $\mathrm{Tc}^{99 \mathrm{~m}}$ scan showed very low $0.2 \%$ homogeneous thyroid uptake (0.6-3.5) with no increased uptake in the nodule. There was a history of mild hirsutism, but testosterone was normal at $0.7 \mathrm{nmol} / \mathrm{L}(0.2-2.7)$ and sex hormone binding globulin (SHBG) was raised at $396 \mathrm{nmol} / \mathrm{L}$ (27-146), as she was on a combined antiandrogenic oral contraceptive. As the surgical removal of the ovarian cyst was essential and urgent, the clinical priority was to treat with carbimazole to render her euthyroid for operation and await the effect on her thyroid function of the surgery and the histology. It was therefore not clinically indicated to perform a pelvic radio iodine scan before surgery. There was therefore a chance of avoiding a radiation dose to the pelvis in this nulliparous patient who was planning conception.

\section{Treatment}

In order to render her euthyroid for surgery, she was started on carbimazole $10 \mathrm{mg}$. After 13 days, TSH increased to $11.9 \mathrm{mU} / \mathrm{L}$, FT4 had increased to $7.7 \mathrm{pmol} / \mathrm{L}$ and FT3

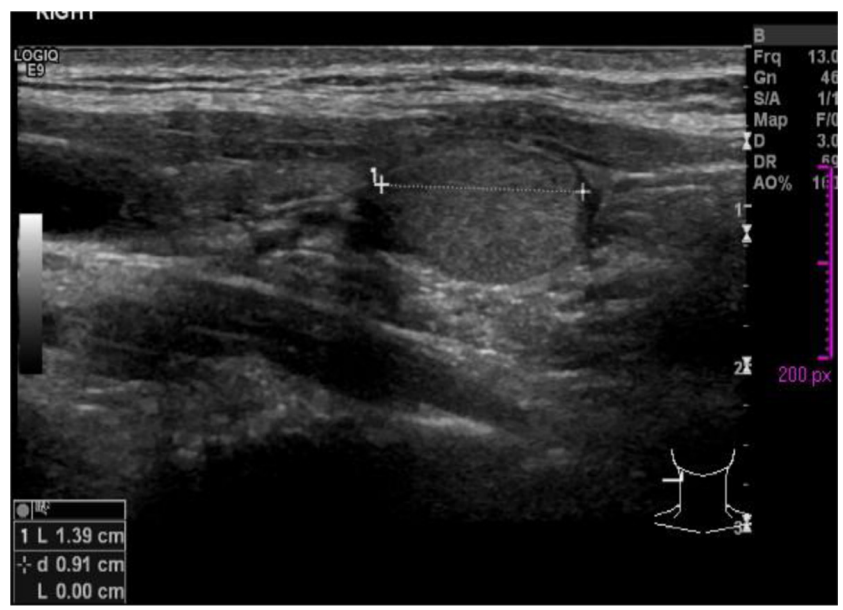

\section{Figure 2}

Thyroid ultrasound showing the solitary right lower lobe thyroid nodule of indeterminate appearance (U3 BTA).

reduced to $3.6 \mathrm{pmol} / \mathrm{L}$, which was safer for surgery. On day 17, a laparoscopic ovarian cystectomy was performed. Carbimazole was reduced to $5 \mathrm{mg}$ and tailed off, see Table 2 .

\section{Outcome and follow-up}

Histology showed Struma Ovarii with the classical benign thyroid follicular appearance (Fig. 3). The specimen was received piecemeal due to laparoscopic removal. One side of the cyst wall included a frond-like appearance. Sections consisted of thyroid tissue with a mixed macrofollicular and microfollicular morphology with colloid containing follicles. There was no penetration of the capsular surface where included. No vascular invasion was present and there were no features of papillary carcinoma. FT4 continued to rise to normal after surgery and after carbimazole was discontinued. The patient was followed up with repeat pelvic ultrasound and thyroid function. All the thyroid function tests before, during and after treatment are presented in Table 2. All her thyroid function tests, TSH, FT3 and FT4 remained normal off any thyroid medication post-treatment. The thyroid function remained normal even 2 years later, when a peritoneal mass was shown to disseminate to a highly differentiated follicular carcinoma. There were multiple small tumours visible on the pelvic peritoneum on scan. At operation, recurrence was noted in the pelvis with multiple small nodules ranging from 1 to $10 \mathrm{~mm}$ anterior to the uterus in the utero-vesical space and confluent disease posterior to the uterus of about $6 \mathrm{~cm}$ diameter involving both pelvis sidewalls, the utero-sacral ligaments and the para-rectal spaces. The peritoneal nodules were all well circumscribed 
Table 2 Thyroid hormone levels before, during and after carbimazole therapy and ovarian cystectomy.

\begin{tabular}{l} 
Carbimazole dose \\
\hline Duration of treatment (days) \\
TSH mU/L \\
FT4 pmol/L \\
FT3 pmol/L
\end{tabular}

\begin{tabular}{c}
\hline Normal range \\
\hline $0.3-3.0$ \\
$10-28$ \\
$4.3-8.1$ \\
\hline
\end{tabular}

\begin{tabular}{c}
\hline Pre-treatment \\
\hline 0 \\
0.06 \\
5.5 \\
6.9 \\
\hline
\end{tabular}

$\begin{array}{r}\hline \mathbf{1 0} \mathbf{~ m g} \\ \hline 10 \\ 0.03 \\ 3.9 \\ 5.2 \\ \hline\end{array}$

\begin{tabular}{c}
\hline $\mathbf{1 0} \mathbf{~ m g}$ \\
\hline 23 \\
11.89 \\
7.7 \\
3.6 \\
\hline
\end{tabular}

\begin{tabular}{l}
\hline $\mathbf{5 ~} \mathbf{~ g}$ \\
\hline 50 \\
8.05 \\
8.7 \\
4.1 \\
\hline
\end{tabular}

\begin{tabular}{l}
\hline $\mathbf{0}$ \\
\hline 75 \\
1.87 \\
9.8 \\
3.7 \\
\hline
\end{tabular}

\begin{tabular}{c}
\hline $\mathbf{0}$ \\
\hline 100 \\
1.73 \\
10.89 \\
4.5 \\
\hline
\end{tabular}

Ovarian cystectomy was performed on day 17

and showed histological features resembling eutopic thyroid tissue with a macrofollicular and microfollicular morphology and no features of papillary carcinoma and therefore the diagnosis of highly differentiated follicular carcinoma was made. It was well recognised that due to its innocuous histology, diagnosis of the latter, formerly known as 'benign strumosis', cannot be made until the neoplasm spreads beyond the ovary. Total thyroidectomy and radioactive iodine-131 therapy was planned.

\section{Discussion}

The finding of the suppressed TSH and low FT4 alone suggested possible hypopituitarism, although usually TSH remains detectable. This was discounted with the finding of the raised FT3. This fitted with her clinical history of the absence of hypopituitary symptoms and 1 year of slight weight loss with hot and sweaty feelings. Another possible cause of these results is factitious T3 self-administration. However, there was no suggestion of drug or T3 abuse. Thyroglobulin assay could have helped to discriminate, but she had positive TgAb which might have caused underestimation. Possible assay interference was discounted using a different assay platform for FT3, FT4 and TSH. Graves' disease was discounted by the

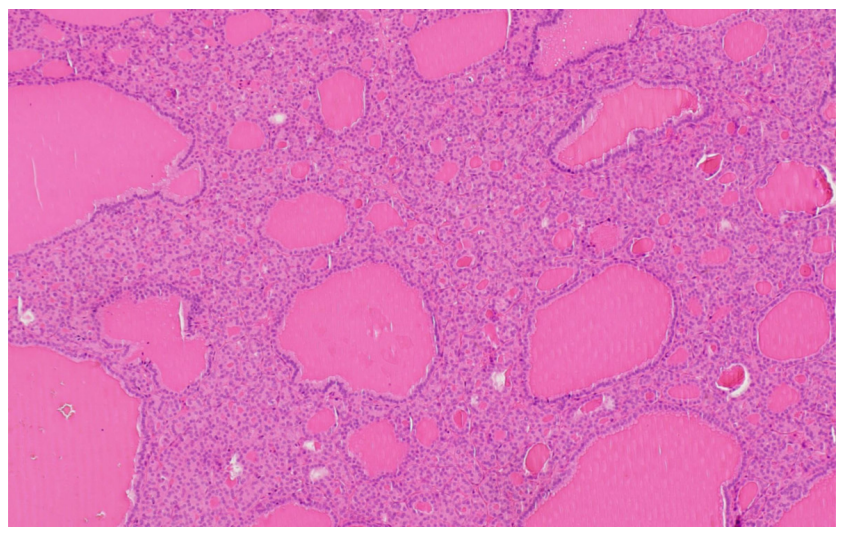

Figure 3

Histology of the left ovarian cyst showing Struma Ovarii. finding of a negative TSHAb, almost undetectable thyroid technetium uptake of $0.2 \%$ and a low thyroid blood flow in the main gland on ultrasound. The TgAb was positive, with some very mild heterogeneous changes on ultrasound and a very low technetium thyroid uptake. Therefore, the toxic phase of autoimmune thyroiditis was a possibility. There was absence of any technetium uptake in the solitary thyroid nodule which discounted the nodule as a cause of the T3 thyrotoxicosis. In addition, all of the other causes mentioned as a possible cause of raised $\mathrm{T} 3$ of thyroid origin would usually be characterised by a T4 level which would either also be raised or in the normal range. The raised FT3 and low FT4 at less than 50\% below the lower limit of normal in this case confirmed TSH suppression due to pure T3 secretion which had the effect of suppressing the thyroid gland T4 production leading to the very low FT4. This combination of results therefore pointed to a T3 production from a source outside the thyroid gland. This was confirmed by the unique paradoxical rise of FT4 in response to carbimazole therapy. When carbimazole was started prior to urgent surgery, the FT3 decreased, as expected, due to the thyroid hormone synthesis blocking effect of carbimazole on any follicular cells actively secreting T3, in this case in the Struma. When the T3 suppression of TSH was removed, the TSH level rose as expected and started to stimulate the thyroid in the normal way. This resulted in the gradual rise in T4 secretion from the thyroid gland and the resulting unique paradoxical rise in the FT4, initially seen with carbimazole, and later with surgical removal of the Struma. The cause of the pure $\mathrm{T} 3$ toxicosis from the tumour may have resulted from overexpression of DIO2 which converted $\mathrm{T} 4$ to $\mathrm{T} 3$ in the tumour. It has been demonstrated that DIO2 is expressed both in normal ovarian tissue (4) and, in addition, in pathological ovarian tumour tissue, most often on bowel metastases (5). The dissemination of the apparently benign appearance of the Struma histology is no surprise, as Struma histology is known to be no guide to the likelihood of malignancy (6) and as dissemination to peritoneal follicular carcinoma has been described up to 26 years after oophorectomy (7). 
All the conventional tests in this case pointed to an excessive T3 secretion from a source outside the thyroid gland. The enlarging ovarian cyst was seen as the probable source if a Struma Ovarii was proven histologically. Previous Struma Ovarii reports resulting in thyrotoxicosis have all described raised or normal combinations of FT3 and FT4. The only previous case of T3 thyrotoxicosis from a Struma was reported by Simkin et al. (3), which described a raised FT3 with the FT4 remaining in the normal range before, during and after treatment. Therefore the Struma was secreting both T4 and T3, otherwise the FT4 would also have been well below the normal range, as in this case.

This is the first report describing the clinical presentation and investigation of a Struma Ovarii shown to be secreting T3 hormone only. This pure secretion of $\mathrm{T} 3$ from an extrathyroidal source resulted in the initial low TSH and FT4, which gave no initial clue as to the possible diagnosis. Only conventional thyroid tests were required to diagnose the extrathyroidal pure T3 secretion, and this finding was confirmed by the rise of FT4 to normal with carbimazole therapy. The unique paradoxical rise of FT4 with carbimazole confirmed the diagnosis which was also proven on histology. The patient subsequently presented 2 years later with peritoneal highly differentiated follicular carcinoma. This was nonsecreting and her thyroid function tests have remained normal. Total thyroidectomy and radioactive iodine- 131 therapy are planned.

\section{Declaration of interest}

The authors declare that there is no conflict of interest that could be perceived as prejudicing the impartiality of the research reported.

\section{Funding}

This research did not receive any specific grant from any funding agency in the public, commercial or not-for-profit sector.

\section{Patient consent}

Signed informed written consent was obtained from the patient and is filed in her clinical notes.

\section{Author contribution statement}

James Prentice was a medical student on attachment to the department of Endocrinology at Croydon University Hospital when the patient was presented at the MDT and did all the data collection and wrote the first drafts of the case. Kate Panter and Thomas Ind were the patient's gynaecological clinicians. Ayoma Attygalle was the histopathologist who reported on the histology of the operation specimen. Malcolm Prentice was the endocrinologist and general medical physician who managed the patient's endocrine and general medical care.

\section{References}

1 Kondi-Pafiti A, Mavrigiannaki P, Grigoriadis Ch, KontogianniKatsarou K, Mellou A, Kleanthis CK \& Liapis A. Monodermal teratomas (struma ovarii). Clinicopathological characteristics of 11 cases and literature review. European Journal of Gynaecological Oncology 201132 657-659.

2 Yoo SC, Chang KH, Lyu MO, Chang SJ, Ryu HS \& Kim HS. Clinical characteristics of struma ovarii. Journal of Gynecologic Oncology 2008 19 135-138. (https://doi.org/10.3802/jgo.2008.19.2.135)

3 Simkin PH, Ramirez LA, Zweizig SL, Afonso SA, Fraire AE, Khan A, Dunn AD, Dunn JT \& Braverman LE. Monomorphic teratoma of the ovary: a rare cause of triiodothyronine toxicosis. Thyroid 19999 949-954. (https://doi.org/10.1089/thy.1999.9.949)

4 Rae MT, Gubbay O, Kostogiannou A, Price D, Critchley HOD \& Hillier SG. Thyroid hormone signaling in human ovarian surface epithelial cells. Journal of Clinical Endocrinology and Metabolism 2007 92 322-327. (https://doi.org/10.1210/jc.2006-1522)

5 Mariani A, Wang C, Oberg AL, Riska SM, Torres M, Kumka J, Multinu F, Sagar G, Roy D, Jung DB, et al. Genes associated with bowel metastases in ovarian cancer. Gynecologic Oncology 2019154 495-504. (https://doi.org/10.1016/j.ygyno.2019.06.010)

6 Shaco-Levy R, Peng RY, Snyder MJ, Osmond GW, Veras E, Bean SM, Bentley RC \& Robboy SJ. Malignant struma ovarii: a blinded study of 86 cases assessing which histologic features correlate with aggressive clinical behavior. Archives of Pathology and Laboratory Medicine 2012 136 172-178. (https://doi.org/10.5858/arpa.2011-0092-OA)

7 Roth LM \& Karseladze AI. Highly differentiated follicular carcinoma arising from struma ovarii: a report of 3 cases, a review of the literature, and a reassessment of so-called peritoneal strumosis. International Journal of Gynecological Pathology 200827 213-222. (https://doi.org/10.1097/PGP.0b013e318158e958)

Received in final form 9 December 2019

Accepted 25 February 2020 